\title{
Compressional acoustic wave generation in microdroplets of water in contact with quartz crystal resonators
}

\author{
L. McKenna ${ }^{\mathrm{a}}$, M.I. Newton ${ }^{\mathrm{a}+}$, G. McHale ${ }^{\mathrm{a}}$, R. Lucklum ${ }^{\mathrm{b}}$ and J. Schroeder ${ }^{\mathrm{b}}$ \\ ${ }^{a}$ Department of Chemistry and Physics, The Nottingham Trent University, \\ Clifton Lane, Nottingham NG11 8NS, UK \\ ${ }^{\mathrm{b}}$ Institute for Micro- and Sensor Systems, Faculty of Electrical Engineering and \\ Information Technology, Otto-von-Guericke-University Magdeburg, P.O.B 4120, D-39016 \\ Magdeburg, Germany
}

\begin{abstract}
Resonating quartz crystals can be used for sensing liquid properties by completely immersing one side of the crystal in a bulk liquid. The in-plane shearing motion of the crystal generates shear waves which are damped by a viscous liquid. Thus only a thin layer of fluid characterised by the penetration depth of the acoustic wave is sensed by a thickness shear mode resonator. Previous studies have shown that the finite lateral extent of the crystal results in the generation of compressional waves, which may cause deviations from the theoretical behavior predicted by a one-dimensional model. In this work, we report on a simultaneous optical and acoustic wave investigation of the quartz crystal resonator response to sessile microdroplets of water, which only wet a localized portion of the surface. The relationship between initial change in frequency and distance from the center of the crystal has been measured for the compressional wave generation regions of the
\end{abstract}


crystal using $2 \mu \mathrm{l}$ and $5 \mu \mathrm{l}$ droplets. For these volumes the initial heights do not represent integer multiples of a half of the acoustic wavelength and so are not expected to initially produce compressional wave resonance. A systematic study of the acoustic response to evaporating microdroplets of water has then been recorded for droplets deposited in the compressional wave generation regions of the crystals whilst simultaneously recording the top and side views by videomicroscopy. The data is compared to theoretically expected values of droplet height for constructive acoustic interference. Results are highly reproducible and there is good correlation between theory and experiment.

\title{
PACS 43.35 Bf
}

Keywords: quartz crystal resonator, QCM, acoustic wave, contact angle, evaporation.

\author{
${ }^{+}$author to whom correspondence should be addressed; \\ electronic mail: michael.newton@ntu.ac.uk \\ telephone: +44 1158483365 \\ fax: +44 1159486636
}




\section{Introduction}

Quartz crystal resonators (QCR) are fabricated by adhering thin electrodes onto the opposing surfaces of AT-cut quartz crystals. Application of an alternating electric field causes the crystal to oscillate in a shearing motion between the electrodes. Changes in the surface mass of the crystal are manifested as a change of the oscillating frequency. Thus the QCR technique lends itself to applications investigating small mass changes at the interface, and the device is termed a quartz crystal microbalance (QCM).

The change of frequency, $\Delta f$, of a QCM device varies directly with mass changes, $\Delta m$, of a thin solid film covering the surface by the relation

$$
\Delta f=\frac{-2 f_{o}^{2} \Delta m}{A \sqrt{\rho_{q} \mu_{q}}}
$$

where $f_{o}$ is the initial resonant frequency of the quartz crystal, $A$ is the active area, defined by the electrode overlap, $\rho_{q}$ is the density of quartz and $\mu_{q}$ is the shear modulus for AT-cut quartz.

A rigid mass, such as a glassy polymer, will oscillate as a unit at the same, reduced, frequency as the QCM. A non-rigid mass, such as water, in contact with the device does not oscillate as a whole in sympathy with the crystal ${ }^{1,2}$. The shear wave disturbance decreases as an exponentially damped cosine function and a boundary layer is conveyed with the surface during the oscillation. The interface between the crystal and adjacent medium is at 
an antinode. The length where the shear wave amplitude reduces to $\mathrm{e}^{-1}$ of the original maximum oscillation amplitude, is defined as the penetration depth, $\delta$,

$$
\delta=\left(\frac{\eta_{L}}{\pi f_{o} \rho_{L}}\right)^{1 / 2}
$$

where $\eta_{L}$ and $\rho_{L}$ are the viscosity and density of the fluid, respectively. The penetration depth for water contacting a crystal operating at $5 \mathrm{MHz}$ is approximately $0.25 \mu \mathrm{m}$. The water within the decay length is effectively attached to the crystal and can be viewed as a mass load which decreases the operating frequency of the device. The change in operating frequency of the QCM for operation with a thick layer of a viscous Newtonian fluid fully covering and immersing one of the electrodes is

$$
\Delta f=-f_{o}^{3 / 2} \sqrt{\frac{\rho_{L} \eta_{L}}{\pi \rho_{q} \mu_{q}}}
$$

The frequency response of a real QCM with its finite lateral dimensions to loading is further complicated because the mass sensitivity across the crystal is not linear. As a consequence of the finite size of the electrodes, the translational disturbance amplitude, and hence sensitivity, is greatest at the center and decreases towards the perimeter in an approximately Gaussian fashion ${ }^{3,4}$. 
Solving the Navier-Stokes equation and the equation of continuity with the assumption of a non-uniform shear flow, as required by the finite electrode size, results in flow normal to the surface ${ }^{3,5}$; the out of plane displacement is around two orders of magnitude smaller than the in-plane displacement. The longitudinal acoustic pressure wave has a wavelength that is dependent upon the acoustic properties of the medium and device frequency. If the path length through the medium is an integer number of half wavelengths, and dissipation of the wave energy into the medium is not too great, a stationary wave may be formed in the liquid cavity. The longitudinal waves may travel a significant distance through the fluid without experiencing great loss. Subsequent reflection at the free liquid surface can result in constructive interference of acoustic waves, it is often termed compressional wave resonance; previous work $^{6}$ has used cavities of $8 \mathrm{~mm}$ and $16 \mathrm{~mm}$ in order to obtain a sharp resonance. Compressional waves may recombine with the shear wave component to influence the apparent mass loading on the crystal and induce a significant change in the crystal's oscillating frequency; in addition to the frequency shift there is also a resistance increase ${ }^{6}$. Detection of compressional waves has been reported previously for fluids enclosed between a resonator and a hard, flat reflecting surface ${ }^{5-7}$; passing the reflector through the fluid results in successive resonator frequency discontinuities which are associated with acoustic interference within the fluid. The free surface of a liquid is also known to act as a reflector of compressional waves. It is therefore common for experiments using quartz crystal resonators with one face immersed fully in a fluid to incorporate a non-plane reflector in the cell design to avoid the problem of compressional wave resonance. 
Quartz crystal resonators have also been used to investigate localised wetting of the electrode surface by small droplets of fluid as well as for investigating solid-liquid interfacial properties using one electrode fully immersed in a bulk liquid. In the application of the quartz crystal technique to droplets the device is essentially used as a sensor of wetted area. The shearing motion of the crystal only senses the droplet to within a penetration depth of the electrode surface and so the droplet can be viewed acoustically as if it was a flat disk on the surface. The change in frequency is then due to the extent to which the droplet wets the crystal electrode. This idea has been used to apply the quartz crystal technique to studies of surfactant superspreading ${ }^{8,9}$, to the measurement of contact angles $^{10}$ and most recently to the study of evaporating droplets of volatile liquids ${ }^{11}$. In this latter study, small discontinuities in the resonant frequency were observed and attributed to compressional wave generation. However, this effect was not investigated in detail and did not include the simultaneous optical observations that are essential if such crystals are to be used for studies of interfacial wetting and contact angle determination.

In this work we report the response of quartz crystal resonators to sessile microdroplets of water using simultaneous frequency measurements and video microscopy. The optical observations include simultaneous side and plan views of the droplets. The general Gaussian relationship between initial change in frequency and distance from the center of the crystal for deposited droplets is investigated, particularly for the compressional wave generation regions of the quartz surface. A systematic study of observations of compressional wave formation in evaporating droplets with relation to both oscillation frequency and physical distortion in the observed side view images of the droplets is then presented. 


\section{Experimental}

A $5 \mathrm{MHz}$ polished quartz crystal with opposing gold electrodes of $2.54 \mathrm{~cm}$ and 1.27 cm diameter (Maxtek Inc. Model SC-501-1) was mounted on fine copper wire using either solder or silver conducting paint. The extended wire electrodes were attached to an oscillator circuit designed to operate at around $5 \mathrm{MHz}$. The oscillator circuit, based on a previously published design ${ }^{12}$, was powered by a stabilised $\pm 5 \mathrm{~V}$ power supply and the operating frequency of the crystal was measured every 250ms by a Hewlett-Packard 53132A Universal Counter and recorded by a personal computer via a GPIB interface. The crystal was mounted on an xyz stage to enable good horizontal alignment. A $0.5 \mu$ l glass syringe (SGC Microsyringe) was mounted obliquely on a stand with vertical and horizontal movement performed by vernier adjustment. The needle was gently curved downwards to allow deposition of distilled and deionised water (Fisher Chemicals) droplets normal to the crystal surface. Video cameras (JVC TK-S350) were used to simultaneously record both side and plan images of the droplets, and real time images were stored on video-cassette for subsequent capture and analysis using a DataTranslations DT3152 frame-grabber card and image processing software. Calibration of the optical system was performed by employing a $1 \mathrm{~mm}$ graticule, with $10 \mu \mathrm{m}$ divisions. The ambient conditions were monitored throughout the experiments using a hygrometer and digital thermometer located close to the crystal. Changes in room temperature did not exceed $2^{\circ} \mathrm{C}$ and changes in humidity did not exceed $2 \%$ either during an experiment or between experiments. The crystal, oscillator and cameras were mounted on an optical table, itself standing on thick rubber feet, to reduce 
extraneous vibration. All sensitive equipment was located in a draft free enclosure. A schematic diagram of the experimental arrangement is shown in Fig. 1.

Compressional wave distribution across a quartz crystal resonator for a fully immersed crystal face has previously been determined experimentally using a hydrophone needle $^{6}$. A typical contour that was measured for the immersed crystals is illustrated in Fig. 2, where the compressional wave formation area is shown as the dark lobes in the $\mathrm{x}$ direction; the center of the electrode is defined as the origin. This was used as a guide for an extensive series of preliminary experiments. Depositing droplets at locations upwards in the $y$-direction on the center $(x=0)$ axis would not cross the compressional wave generation regions, whereas droplets moving across the $x$-axis with $y=0$ would cross these regions. Following the preliminary investigations, ten series of measurements for droplet volumes of $0.2 \mu \mathrm{l}$ and $0.5 \mu \mathrm{l}$ were performed; the $0.2 \mu \mathrm{l}$ droplets produce a wetted area of approximately $0.6 \mathrm{~mm}^{2}$ and the $0.5 \mu \mathrm{l}$ approximately $1.2 \mathrm{~mm}^{2}$. Each series consisted of depositing a droplet whilst continually recording the oscillation frequency of the crystal and video recording the side and plan views of the droplet. After complete evaporation, the next droplet was deposited $1 \mathrm{~mm}$ further along the axis and the measurement repeated. Measurements were made along both the $x$ and $y$ axes of the crystal, passing through the center in each case.

\section{Results and Discussion}

The deposition of a droplet of water on a crystal results in a sudden reduction of the oscillating frequency. Progressively larger droplets deposited at the same location produce progressively greater initial frequency reductions. Changing the position at which the 
droplet is deposited on the crystal for similarly sized droplets also results in incrementally greater frequency reductions as the position of deposition approaches the center of the electrode. This is shown for a series of $0.5 \mu \mathrm{l}$ and $0.2 \mu \mathrm{l}$ droplets in Fig. 3, where the fitted curves are Gaussian profiles. The droplet position is moving from the center along the $x$ direction shown Fig. 2 and hence represents moving across the compressional wave generation lobes. For these droplet volumes, the initial heights did not represent integer multiples of a half of the acoustic wavelength and so would not be expected to produce compressional wave resonance.

When a droplet of water is sufficiently small, the influence of gravity becomes negligible and it forms a spherical cap shape on the crystal surface; its subsequent change of shape as it evaporates has been reported in the literature ${ }^{13,14}$. If the droplet of water has an initial contact angle of less than 90 degrees, then as the droplet evaporates the angle will initially decrease approximately linearly in time whilst maintaining a constant contact radius. Eventually the radius will begin to shrink rapidly, but the regime of constant contact area holds for the majority of the evaporation time. When deposited on the resonating quartz crystal, provided the droplet height is greater than the penetration depth of the acoustic wave into the fluid, the constant contact area of the droplet as it evaporates will mean that little change in frequency should occur. As the contact radius rapidly contracts a monotonic recovery of the oscillating frequency to the value prior to droplet deposition should be observed. This behavior of the device frequency is clearly demonstrated in the upper line of Fig. 4. In this case a $0.5 \mu \mathrm{l}$ water droplet was deposited $1 \mathrm{~mm}$ from the crystal center, but outside the compressional lobes. The initial fall in frequency as the droplet was 
deposited was followed by a relatively constant frequency region for 350 seconds whilst the contact area remained unchanged. The contraction of the radius then began and as the film finally evaporated, the crystal returned to the original frequency. The described behavior of the contact angle, drop height and wetted area were confirmed by the simultaneous side and plan views of the droplet.

The lower curve shown in Fig. 4 is for a $0.5 \mu \mathrm{l}$ water droplet deposited $1 \mathrm{~mm}$ from the crystal center within the periphery of one of the compressional lobes. The initial fall in frequency as the droplet is deposited is similar to the upper curve, but instead of a period of constant frequency while the contact area is constant, discontinuities in the frequency are clearly observable. These discontinuities can result in frequency shifts larger than the initial reduction in frequency due to droplet deposition and cannot therefore be regarded as minor perturbations of the shear wave response; the sample time of $250 \mathrm{~ms}$ is unlikely to be sufficiently short to accurately record the true maximum in the frequency deviation. The eventual contraction of the contact radius gives rise to an increase in the frequency as the wetted area decreases, but even in this period significant discontinuities continue to be observed. As previously, when the film finally evaporated the crystal returned to the original frequency. The initial operating frequencies for both curves in Fig. 4 are identical, but the upper curve has been displaced by $85 \mathrm{~Hz}$ for clarity.

The frequency versus time data of many separate droplet depositions were analyzed in order to locate the frequency discontinuities which, we suggest are due to compressional wave resonance; at these points the optical profiles were investigated in detail. For many of 
the frequency discontinuities significant droplet distortion was observed in the side view images. An example is shown in Fig. 5 where the upper image shows the drop 2 seconds before compressional wave resonance (peak in the acoustic discontinuity) and the lower image shows the same drop during the compressional wave resonance; these images show the drop and its reflection in the substrate. The most striking feature is the distortion and reduction in sharpness of the drop image at its apex, causing the gray area as indicated by the arrow in Fig.5. Most observed optical distortions were coincident with significant $(>10 \mathrm{~Hz})$ frequency discontinuities. However, some strong frequency response peaks did not coincide with image distortion and were the result of contraction of the edges of the droplets; these could be identified and discounted due to observations from the camera monitoring the top view of the droplet.

For an electrode that is completely immersed by liquid of uniform depth, and where the free liquid surface is parallel to the electrode, we can predict the liquid depth for compressional wave resonance from the wavelength defined by ${ }^{4} v=f \lambda$ where $v$ is the wave velocity, $f$ is the oscillating frequency and $\lambda$ is the wavelength. The height, $h$, of the liquid cavity, for constructive interference, is $h=n v / 2 f$ where $n$ is an integer and the factor of two has introduced to acknowledge the wavelength is the return path between the crystal and the liquid surface. For droplets we suggest that the most likely resonant cavity will be formed at the center of the area wetted by the droplet. This location corresponds to the apex of the droplet, which is parallel to the electrode surface. Fig 6 shows the measured droplet height for the image frames that display significant optical distortions plotted against the theoretical height that would be expected for compressional wave resonance. Fig. 7 shows 
the droplet heights that exhibited significant frequency discontinuities plotted against the theoretical height that would be expected for compressional wave resonance. The coincidence, and reproducibility, of experimentally determined droplet heights measured from the images compares well with the expected compressional wave heights.

Whilst Fig. 6 and 7 support the idea that even small droplets can act as resonant cavities for compressional wave resonance, it should be noted that in many of the experiments there are theoretically predicted heights that favour compressional wave resonance where no discontinuities in the optical or acoustic data are apparent. In our experiments where, say, we would expect a series of four discontinuities during the evaporation of any single drop we often only observed three. It is not clear why some resonances were missing, but it may be attributable to non-normal reflections of the acoustic wave energy from the water-air interface due to droplet distorting phenomena, such as pinning of the areal circumference to the crystal before relaxation via 'snapping' of the attachment. It has been previously shown ${ }^{6}$ that a disalignment of only a couple of degrees from the normal can destroy compressional wave resonance. The compressional wave formations observed in our work occur at multiples of half wavelengths above the crystal surface. The greatest propensity, however, is to exhibit interference behavior at heights of $\lambda / 2$ and $\lambda$ above the QCM surface. This is clearly shown in Fig. 8 where the relative occurrence of acoustic discontinuities in the series of experiments is shown by the solid bars and the open bars show the relative occurrence of optical distortions. A possible explaination for this may be that the lower droplet profiles present a greater analogy to plane reflectors than the taller droplets, for the same fixed contact area. An alternative 
explaination is that the greater the height of liquid, the sharper the resonance as previously reported $^{6}$ hence the sample time of $250 \mathrm{~ms}$ may have been too slow to register such rapid frequency discontinuities.

\section{Conclusion}

The effect of small droplets of water on the frequency response of quartz crystal resonators has been systematically investigated. The approximately Gaussian sensitivity of the crystal surface due to the finite size of the electrodes has been confirmed in the response to small droplets of water. However, significant discontinuities in the frequency response have also been observed when droplets are deposited in specific locations known to favor compressional wave generation. Simultaneous video microscopy of both the side and plan profiles of droplets has been used to correlate frequency discontinuities with characteristic sizes of droplets. Data has been analyzed to compare theoretically expected values of droplet height where constructive acoustic interference might be expected to occur. The experimental results have been shown to be reproducible and offer good correlation between theory and experiment and indicate that small sessile droplets can act as resonant cavities for the formation of compressional waves. Frequency changes due to such effects can far exceed that expected from the shear wave damping induced by the electrode area wetted by the droplet.

\section{Acknowledgements}

The authors would like to thank The British Council and the DAAD for financial support. 


\section{References}

${ }^{1}$ S. Bruckenstein and M. Shay, Electrochim. Acta, 30, 1295 (1985)

${ }^{2}$ K.K. Kanazawa and G. J. Gordon, Anal. Chem., 57, 1770 (1985)

${ }^{3}$ B.A. Martin and H.E. Hager, J. Appl. Phys., 65, 2627 (1989)

${ }^{4}$ F. Josse, Y. Lee, S.J. Martin and R.W. Cernosek, Anal. Chem., 70, 237 (1998)

${ }^{5}$ T.W. Schneider and S.J. Martin, Anal. Chem., 67, 3324 (1995)

${ }^{6}$ R. Lucklum, S. Schranz, C. Behling, F. Eichelbaum and P. Hauptmann. Sensors and Actuators A, 60, 40 (1997)

${ }^{7}$ Z. Lin and M.D. Ward, Anal. Chem., 67, 685 (1995)

${ }^{8}$ Z.X. Lin, R.M. Hill, H.T. Davis and M.D. Ward, Langmuir, 10, 4060 (1994)

${ }^{9}$ Z.X. Lin, T. Stoebe, R.M. Hill, H.T. Davis and M.D. Ward, 12, 345 (1996)

${ }^{10}$ Z. Lin and M.D. Ward, Anal. Chem., 68, 1285 (1996)

${ }^{11}$ M.J. Joyce, P. Todaro, R. Penfold, S.N. Port, J.A.W. May, C. Barnes and A.J. Peyton, Langmuir, 8, 4024 (2000)

${ }^{12}$ F. Eichelbaum, R.Borngraeber, J. Schroeder, R. Lucklum and P. Hauptmann, Rev. Sci. Inst., 70, 2537 (1999)

${ }^{13}$ S.M. Rowan, M.I. Newton and G. McHale, J. Phys. Chem., 99, 13268 (1995)

${ }^{14}$ S.M. Rowan, G. McHale, M.I. Newton and M. Toorneman, J. Phys. Chem., B 101, 1266 (1997) 


\section{Figure Captions}

Figure 1. Schematic diagram of the experimental arrangement.

Figure 2. The electrode arrangement for a quartz crystal. The regions of a quartz crystal in which compressional waves are known to be generated are shown as the dark lobes (from reference 6).

Figure 3. Initial frequency change as a function of distance from the center of the crystal for droplets of $0.2 \mu$ l (triangles) and $0.5 \mu$ l (circles) deposited across the region for compressional wave generation; the solid lines show a Gaussian fit to the data.

Figure 4. The oscillation frequency shown as a function of time for $0.5 \mu$ l droplets of water deposited at 30 seconds. The lower line shows data for a droplet deposited $1 \mathrm{~mm}$ from the center in a compressional lobe. The upper line shows data for a droplet at the same distance from the center, but outside the compressional lobes (with an offset of $85 \mathrm{~Hz}$ ).

Figure 5. Images of water droplets prior to (upper image) and during (lower image) the discontinuity in the acoustic signal. An image distortion can be observed at the apex of the drop in the lower image corresponding to the compressional wave resonance. 
Figure 6. The measured droplet heights for images showing significant optical distortion plotted against the theoretical height for compressional wave resonance.

Figure 7. The measured droplet heights for images coincident with acoustic discontinuities plotted against the theoretical height for compressional wave resonance.

Figure 8. The percentage of experiments in which the acoustic discontinuities (solid bars) and optical distortions (open bars) were observed, shown for droplet heights in integer number of quarter wavelengths. 
Figure 1. McKenna et al Journal of Applied Physics

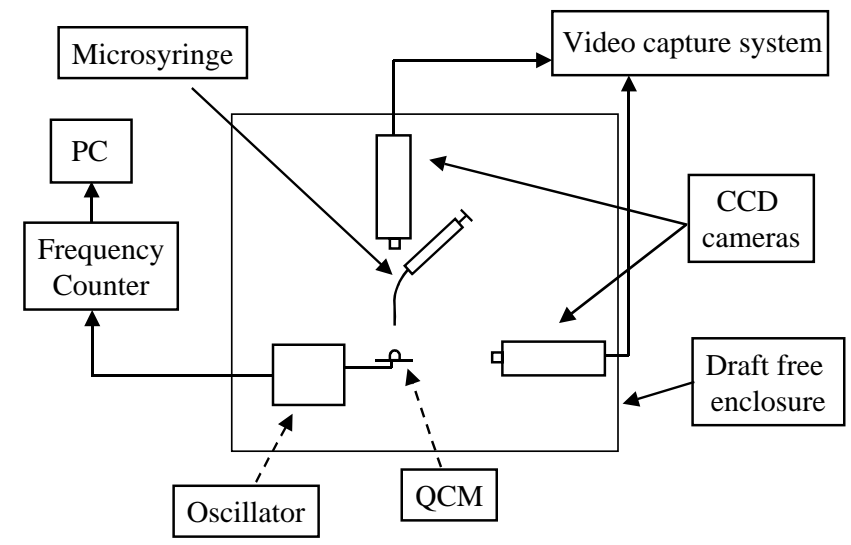


Figure 2 
Figure 3 McKenna et al Journal of Applied Physics

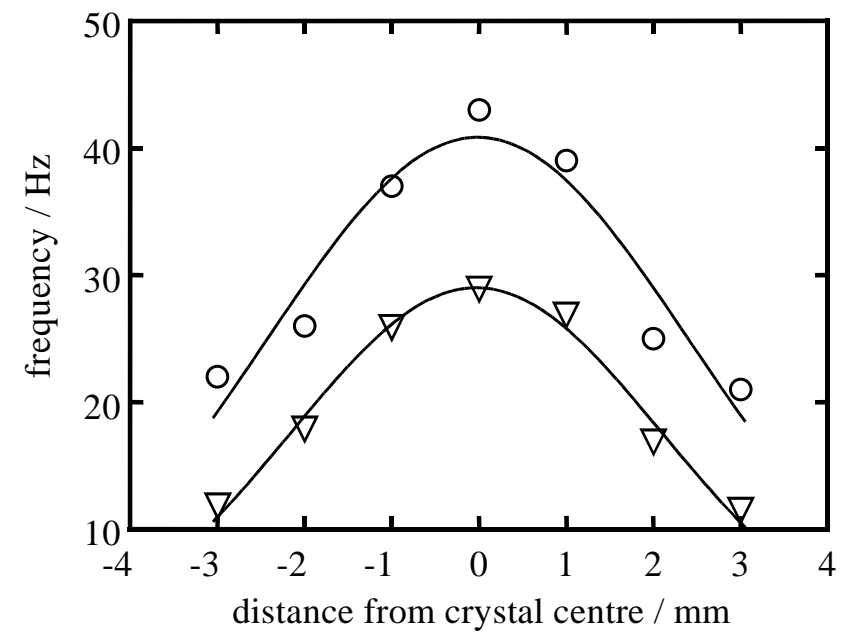


Figure 4 McKenna et al Journal of Applied Physics

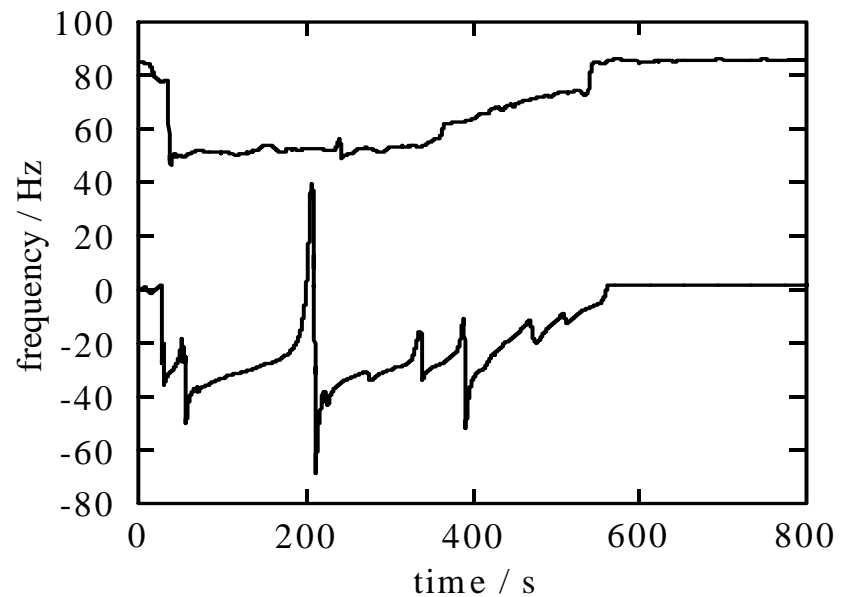


Figure 5 McKenna et al Journal of Applied Physics
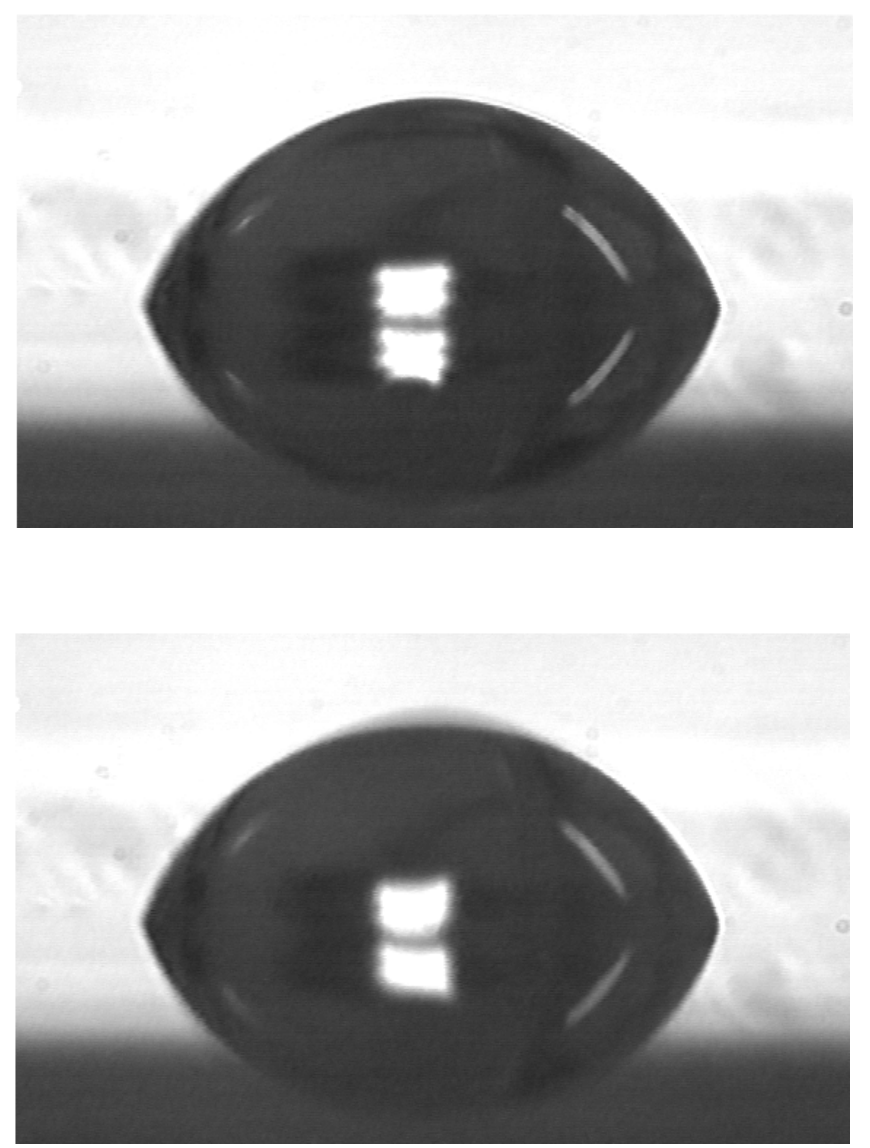
Figure $6 \quad$ McKenna et al Journal of Applied Physics

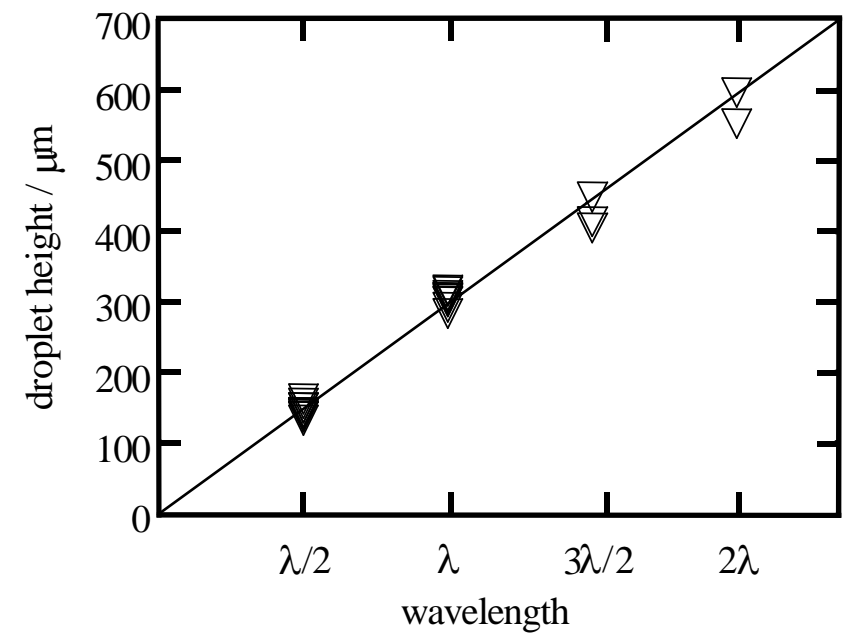


Figure $7 \quad$ McKenna et al Journal of Applied Physics

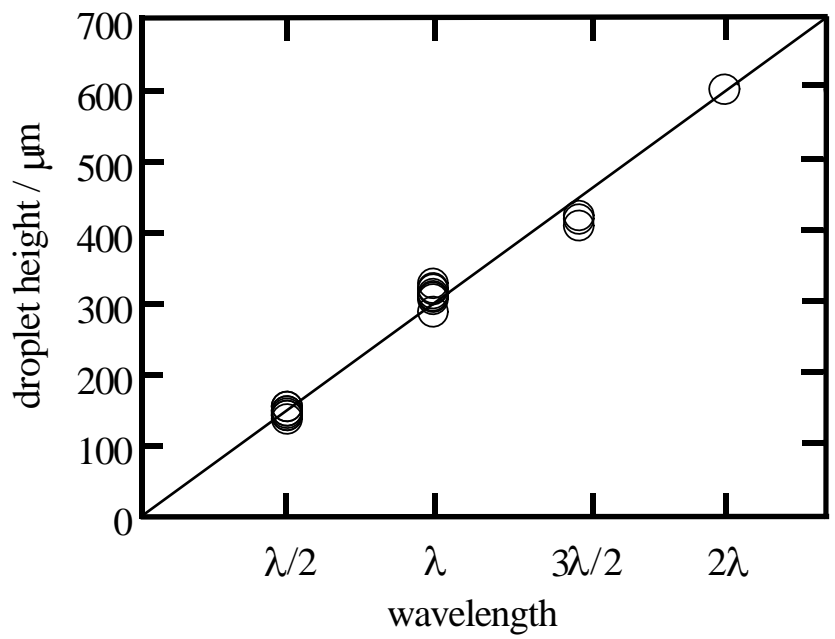


Figure 8 McKenna et al Journal of Applied Physics

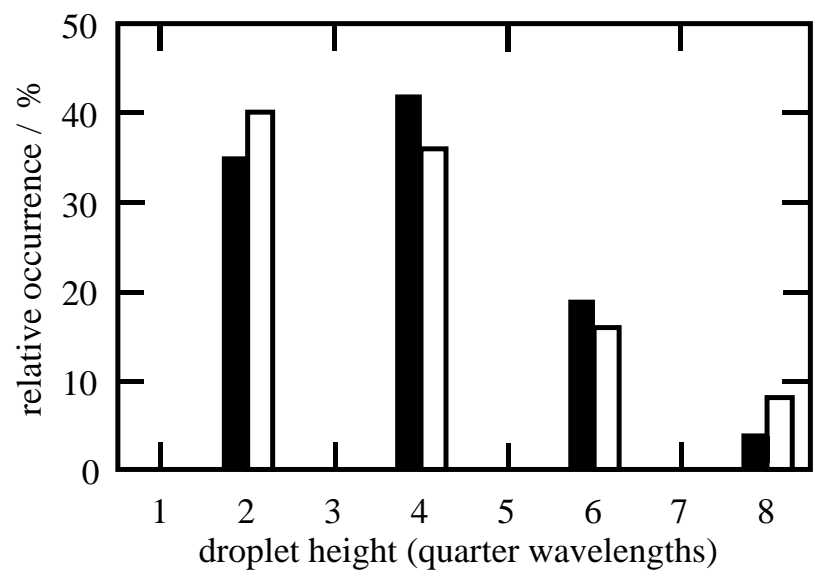

